\title{
Chest CT Findings of Coronavirus Disease 2019 (COVID-19)
}

\author{
Wu Cai, Junlin Yang, Guohua Fan, Liang Xu, Bo Zhang and Rong Liu \\ Department of Radiology, The Second Affiliated Hospital of Soochow University, Suzhou, China
}

\begin{abstract}
The aim of this study was to investigate the chest CT findings of coronavirus disease 2019 (COVID-19) from January to February 2020. CT features of six cases, confirmed by reverse transcription polymerase chain reaction (RT-PCR) with COVID-19 disease, were evaluated. Of the six patients, one patient had normal scan. One patient had ground glass opacity only, while the rest four patients mainly had ground glass opacity accompanied by consolidation. Moreover, air bronchogram, crazy paving and reversed halo sign could be seen in 5, 3 and 1 patient, respectively. Lesions in three patients were only located in the peripheral area, while of two patients, were mainly located in the peripheral area with involvement of the center along with bronchovascular bundles. While one patient had enlarged mediastinal nodes, no one had pleural effusion. CT can be used as the first choice for early diagnosis of COVID-19 pneumonia.
\end{abstract}

Key Words: Pneumonia, Lung CT scan, COVID-19, Coronavirus infection.

How to cite this article: Cai W, Yang J, Fan G, Xu L, Zhang B, Liu R. Chest CT Findings of Coronavirus Disease 2019 (COVID-19). J Coll Physicians Surg Pak 2020; 30(JCPSPCR):CR53-CR55 https://doi.org/10.29271/jcpsp.2020.JCPSPCR.CR53.

Since December 2019, an increasing number of cases of pneumonia infected with coronavirus disease 2019 (COVID-19), started from the Huanan seafood wholesale market have been identified in Wuhan, Hubei province, China. ${ }^{1}$ With the rapid spread of the disease resulted from human-to-human transmission, mainly by respiratory droplets and close contact, COVID-19 cases have been reported in other Chinese cities and around the world, which caused a global health emergency. By May 10, 2020, a total of 84,430 confirmed cases and 4,643 deaths in China, 4,099,410 confirmed cases and 280,512 deaths abroad have been reported. Recent investigations reported that early detection, diagnosis and subsequent early isolation and treatment of COVID-19 are crucial to the patient's good prognosis. $^{2}$ In the clinic, the common symptoms of COVID-19 are fever, nonproductive cough, dyspnea, myalgia and fatigue. However, the incubation period is 1-14 days, mainly 3-7 days. Nowadays, the gold standard for diagnosis of COVID-19 is the viral nucleic acid detection in swabs, sputum, lower respiratory tract secretions or blood. Nevertheless, owing to the suboptimal sensitivity of COVID-19 nucleic acid kits, suspected cases with false-negative results still remain an enormous challenge.

Correspondence to: Guohua Fan, Department of Radiology, The Second Affiliated Hospital of Soochow University, San Xiang Road No. 1055, Suzhou, Jiangsu 215004, China

E-mail: fangh22@yeah.net

Received: May 11, 2020; Revised: June 18, 2020;

Accepted: July 03, 2020

DOI: https://doi.org/10.29271/jcpsp.2020.JCPSPCR.CR53
Furthermore, some studies reported chest CT imaging demonstrated earlier than clinical symptoms, especially in the incubation period. ${ }^{3}$ Hence, CT imaging is of vital importance for early detection and diagnosis of COVID-19. Hitherto, there are a few investigations of CT imaging of COVID-19. In the current study, chest CT images of six confirmed patients were enrolled to investigate the CT findings.

Six reverse transcription polymerase chain reaction (RT-PCR) confirmed-patients with COVID-19, from January 26, 2020 to February 13, 2020 at the Second Affiliated Hospital of Soochow University, were recruited. The study was conducted after approval from the Hospital Ethical Committee. All patients were examined on a 256-section CT system (Brilliance iCT; Philips Healthcare, Cleveland, OH, USA) in the supine position with scanning of the entire chest without contrast in caudio-cranial direction with $0.67 \mathrm{~mm}$ slice thickness.

The CT images were evaluated for the presence of ground glass opacity, consolidation or mixed pattern; air bronchogram, reversed halo sign and crazy paving sign; peripheral, central or combined distribution; location and number of involved lobes and single or multiple lesions within each involved lobe; enlarged mediastinal nodes and pleural effusion.

Among the six patients, the male to female ratio was $2: 1$, mean age was $42.8 \pm 14.2$ years, ranging from 26 to 67 years. Four patients had a recent travel to Wuhan or nearby; and one patient had a close contact with confirmed patient; whereas, one patient had unknown exposure history. Five patients had clinical symptoms of fever and cough, while one patent had myalgia and diarrhea. The leukocyte count was normal in three patients, while decreased and increased in two and one 
patients, respectively. The lymphocyte count was normal in two patients, while decreased in four patients. The C-reactive protein was normal only in one patient, while increased in rest of five patients. The liver enzyme was normal in three patients, while increased in rest of three patients. The lactate dehydrogenases of all the six patients were increased.

Among the six patients, one patient showed no abnormal findings in chest CT imaging (Table I). One patient only had ground glass opacity (Figure 1A), while another four patients mainly had ground glass opacityaccompanied by partial consolidation (Figure 1B and 1C). Moreover, air bronchogram without mucus plug could be seen in five patients (Figure $1 A$ ), one patient had reversed halo sign (Figure 1C), and three patients demonstrated crazy paving sign (Figure 1B-D). These lesions were located in the peripheral area under the pleura in three patients (Figure $1 \mathrm{~A}-\mathrm{C}$ ), while mainly located in the peripheral area with involvement of the centre along bronchovascular bundles in two patients (Figure 1D-G). One patient had three lobes affected excepting right upper and middle lobe, while four patients had involvement of all the five lobes (Figure 1D-G). Only a single patient had single lesion within right upper lobe; whereas, the rest of the affected lobes demonstrated multiple lesions (Figure 1D-G). One patient had enlarged mediastinal nodes (Figure $1 \mathrm{H}$ ); and no one had pleural effusion.

Table I: CT findings of six patients.

\begin{tabular}{|c|c|}
\hline Ground glass opacity and consolidation & Numbers (\%) \\
\hline Presence of ground glass opacity & $1(16.7 \%)$ \\
\hline Presence of ground glass opacity and consolidation & $4(66.6 \%)$ \\
\hline Reversed halo sign & $1(16.7 \%)$ \\
\hline Crazy paving sign & $3(50.0 \%)$ \\
\hline Air bronchogram & $5(83.3 \%)$ \\
\hline \multicolumn{2}{|l|}{ Number of lobes involved } \\
\hline Single lobe & $0(0.0 \%)$ \\
\hline Two lobes & $0(0.0 \%)$ \\
\hline Three lobes & $1(16.7 \%)$ \\
\hline Four lobes & $0(0.0 \%)$ \\
\hline Five lobes & $4(66.6 \%)$ \\
\hline \multicolumn{2}{|l|}{ Frequency and lesions of lobe involvement } \\
\hline Right upper lobe & $4(66.6 \%)$ \\
\hline Single lesion & $1(16.7 \%)$ \\
\hline Multiple lesions & $3(50.0 \%)$ \\
\hline Right middle lobe (multiple lesions) & $4(66.6 \%)$ \\
\hline Right lower lobe (multiple lesions) & $5(83.3 \%)$ \\
\hline Left upper lobe (multiple lesions) & $5(83.3 \%)$ \\
\hline Left lower lobe (multiple lesions) & $5(83.3 \%)$ \\
\hline \multicolumn{2}{|l|}{ Distribution } \\
\hline Peripheral & $3(50.0 \%)$ \\
\hline Peripheral involving central & $2(33.3 \%)$ \\
\hline \multicolumn{2}{|l|}{ Other features } \\
\hline Enlarged mediastinal nodes & $1(16.7 \%)$ \\
\hline Pleural effusion & $0(0.0 \%)$ \\
\hline Normal scan & $1(16.7 \%)$ \\
\hline
\end{tabular}

From December 2019, the outbreak of an epidemical pneumonia in Wuhan, China, was ultimately found to be caused by a new BetaCoronavirus named as COVID-19 by the World Health
Organization. This is the new member of large RNA coronavirus family that includes severe acute respiratory syndrome coronavirus (SARS-Cov), Middle-East respiratory syndrome coronavirus (MERS-Cov), and whose genome sequence is $85 \%$ similar to SARS-Cov, both can cause severe respiratory syndrome in humans. ${ }^{2}$ Therefore, early detection and diagnosis of COVID-19 is vital for disease treatment and control. Owing to its high sensitivity and convenience, chest CT imaging has become the primary tool for diagnosis of COVID-19 as compared with initial RT-PCR from swab samples because of limitations of sample collection and transportation and kit performance. ${ }^{3}$ Thus, in this study, six confirmed patients were searched to analyse CT features to detect and diagnose at early age for determining hospitalization and isolation.

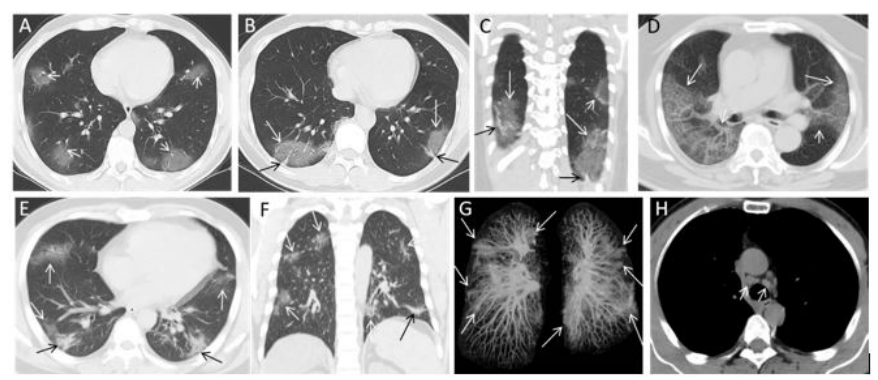

Figure 1: Chest CT abnormal findings of the enrolled patients with COVID-19.

A: Multiple ground glass opacities with air bronchogram (short white arrows) distributed along peripheral subpleural regions; B,C: Crazy paving sign manifested by bilateral lower lobes ground glass opacities and interlobular septal thickening (long white arrows) accompanied by partial consolidation (long black arrows); C: Ground glass opacity with reversed halo sign (short white arrow) in the subpleural area of left lower lobe; D: Multiple ground glass opacities with crazy paving sign distributed along peripheral subpleural regions (long white arrows) and involvement of the center along bronchovascular bundles (short white arrows); E-G: Multiple ground glass opacities accompanied by partial consolidation diffused in the bilateral lobes; $\mathrm{H}$ : Mediastinal window demonstrated enlarged mediastinal nodes (short white arrows).

There were reports on CT imaging of COVID-19 in the past several months. ${ }^{2,4} \mathrm{CT}$ findings overlapped with other viral pneumonia such as SARS, MERS etc. Nevertheless, COVID-19 still has its characteristic CT features. In this study, only one patient had three lobes including bilateral lower lobes affected, while four patients had all lobes affected. Furthermore, multiple lesions were demonstrated in all involved lobes except single lesion in a right upper lobe. Meanwhile, lesions distributed peripheral area under the pleura, and two patients involved the centre along bronchovascular bundles, but lesions still mainly located in the peripheral area. The physiopathological mechanism for above-mentioned imaging characteristics is still unsure. However, it is speculated that the release storm of inflammatory cellular cytokines plays a vital role in the COVID-19, and the diameter of lower lobe bronchus is relatively larger than that of upper and middle lobe. Moreover, the Coronavirus's targeted cell may locate in the epithelium of respiratory bronchiole and pulmonary alveoli. In this study, ground glass opacity in five patients and accompanied by partial consolidation in four patients were observed. These five patients came to the hospital at early 
stage of COVID-19, when different degree of diffuse pulmonary alveoli injury with hyaline membrane formation, but little of exudation and edema in pulmonary alveoli. Partial consolidation could be seen in the center of some ground glass opacity resulted from the increase of inflammatory exudation in pulmonary alveoli. Then, with the progression of pulmonary interstitial infection and thickening, crazy-paving sign revealed in three patients. Fibrous stripe was rare in these six patients because they were all at the early stage of COVID-19, which often demonstrated at the recovery stage except combined with other primary pulmonary diseases. Air bronchogram without mucus plug seen in five patients could account for the symptom of nonproductive cough.

When the viruses invaded the lymphatic system, the mediastinal node enlarged through the pulmonary lymphatic drainage. Thus, one patient had enlarged mediastinal nodes. One patient had no abnormal findings in chest CT in the study, it is speculated that COVID-19 has an incubation period of 1-14 days, and symptoms occur prior to the imaging manifestations.

CT features of viral pneumonia have been well known. ${ }^{5}$ However, the features overlap among various viral organisms. COVID-19 should be differentiated from other viral pneumonia such as SARS, MERS, influenza virus, adenovirus etc. Especially for SARS and MERS, they have many similar CT features to COVID-19 because the causative viruses belong to the same family. Nevertheless, definite epidemical exposure history is vital for COVID-19. Meanwhile, COVID-19 should also be differentiated from other microorganisms including bacteria, chlamydia and mycoplasma.

In summary, CT findings of COVID-19 are mostly multiple ground glass opacities in the bilateral peripheral subpleural areas or involvement of center along bronchovascular bundles accompanied by partial consolidation, air bronchogram, reversed halo and crazy paving sign as reported by others. The presence of enlarged mediastinal nodes is an unusual finding.

\section{ETHICALAPPROVAL:}

The study was conducted after obtaining approval from the Hospital Ethical Committee.

\section{CONFLICT OF INTEREST:}

Authors declared no conflict of interest.

\section{AUTHORS' CONTRIBUTION:}

WC, GF: Designed the study, interpreted data and drafted the manuscript.

JY, RL: Collected data and edited the manuscript.

$B Z, L X$ : Analysed CT images and revised critically the manuscript.

\section{FUNDING:}

Suzhou Municipal Science and Technology Project (SYS20 20027, SYS2018057 and SYSD2016088) and Pre-research Foundation of Second Affiliated Hospital of Soochow University (SDFEYBS1904 and SDFEYQN1816).

\section{REFERENCES}

1. Li Q, Guan X, Wu P, Wang X, Zhou L, Tong Y, et al. Early transmission dynamics in Wuhan, China, of novel coronavirus-infected pneumonia. $N$ Engl J Med 2020; 382:1199-207.

2. Chen N, Zhou M, Dong X, Qu J, Gong F, Han Y, et al. Epidemiological and clinical characteristics of 99 cases of 2019 novel coronavirus pneumonia in Wuhan, China: A descriptive study. Lancet 2020; 395:507-13.

3. Huang $P$, Liu T, Huang L, Liu H, Lei M, Xu W, et al. Use of chest CT in combin- ation with negative RT-PCR assay for the 2019 novel coronavirus but high clinical suspicion. Radiology 2020; 295:22-3.

4. Fang $Y$, Zhang $H, X u Y, X i e ~ J$, Pang $P$, Ji W. CT manifestations of two cases of 2019 novel coronavirus (2019nCoV) pneumonia. Radiology 2020; 295:208-9.

5. Koo HJ, Lim S, Choe J, Choi SH, Sung H, Do KH. Radiographic and CT features of viral pneumonia. Radiographics 2018; 38:719-39. 